\title{
MARKETING TURÍSTICO PARA MILLENNIALS
}

\author{
TOURIST MARKETING FOR MILLENNIALS
}

Betsy Lissett Cornelio Vázquez* María Cruz Cuevas Álvarez**

*Estudiante de la División Académica de Ciencias Económico Administrativas de la Universidad Juárez Autónoma de Tabasco. ORCID: https://orcid.org/0000-0002-0323-2216.

**Doctora en Administración Educativa. Profesora Investigadora de la División Académica de Ciencias Económico Administrativas de la Universidad Juárez Autónoma de Tabasco. Email: maria.cuevas@ujat.mx. ORCID: https://orcid.org/0000-0003-2349-5344.

Dirección para recibir correspondencia: betsy.lcv@gmail.com

Fecha de recibido: 30 de noviembre de 2018

Fecha de aceptación: 12 de enero de 2019

DOI: https://doi.org/10.19136/hitos.a25n71.3224

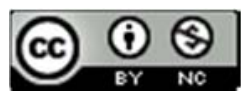

CORNELIO-VÁZQUEZ B. L., CUEVAS ÁLVAREZ M. C. 
MARKETING TURÍSTICO PARA MILLENNIALS.

\section{RESUMEN}

OBJETIVO: Conocer los medios por los cuales los millennials acceden a la búsqueda de información, así como la preferencia en la elección de un destino turístico.

MATERIAL Y MÉTODO: Se realizó través del método inductivo con las técnicas cualitativas de revisión documental, tabla de contenido y grupo de enfoque con una selección intencional de 10 participantes entre los 20 y 35 años de edad de diversas áreas del conocimiento y del sector productivo.

RESULTADOS: Diversos estudios se han realizado sobre el turista tradicional, así como las estrategias implementadas para la promoción de destinos turísticos; sin embargo, se observa un vacío en la literatura sobre el turista millennial, así como sobre las preferencias en lo que a destinos turísticos se refiere. Este tipo de turista realiza su búsqueda y reservaciones haciendo uso del internet y redes sociales; gustan de destinos de playa nacionales e internacionales, cruceros y actividades donde haya una cercanía con la cultura o un gusto extrapolado hacia la modernidad, con una característica en particular: experiencias turísticas. Por ser una generación que pierde el interés rápidamente, sus destinos y preferencias cambian drásticamente, causando que las tendencias tengan una durabilidad menor que en años anteriores.

CONCLUSIONES: Se concluye entonces, que la oferta más apropiada para este segmento sea la propuesta de Destinos Turísticos Inteligentes (DTI) y que los proveedores de servicio lleven sus esfuerzos mercadológicos hacia esa tendencia.

PALABRAS CLAVE: Millennial. Preferencias. Destinos Turísticos Inteligentes. 


\section{MARKETING TURÍSTICO PARA MILLENNIALS.}

\section{ABSTRACT}

OBJECTIVE: To know the means by which millennials access to the information search, as well as their preference in the choice of a tourist destination.

MATERIAL AND METHOD: It was carried out through the inductive method with the qualitative techniques of documentary review, table of contents and focus group with an intentional selection of 10 participants between 20 and 35 years of age from different areas of knowledge in the productive sector.

RESULTS: Various studies have been carried out on the traditional tourist, as well as the strategies implemented for the promotion of tourist destinations; however, there is a gap in the literature on the millennial tourist, as well as on their preferences in terms of tourist destinations. This type of tourist conduct their search and reservations using the Internet and social networks; they like national and international beach destinations, cruises and activities where there is a closeness to culture or an extrapolated taste towards modernity, with a particular feature: tourist experiences. As a generation that quickly loses interest, their destinations and preferences change drastically, causing shorter validity period trends compared to previous years.

CONCLUSIONS: It is concluded that the most appropriate offer for this segment is the proposal of Intelligent Tourist Destinations (ITD) plus, that the service providers apply their marketing efforts towards this trend.

KEY WORDS: Millennial. Preferences. Intelligent Tourist Destinations. 


\section{MARKETING TURÍSTICO PARA MILLENNIALS.}

\section{INTRODUCCIÓN}

El sector turístico es una de las grandes fuentes de ingreso en México, tanto por el turismo extranjero como por el turismo local, el cual con el paso del tiempo ha incrementado gradualmente. Dentro de los principales turistas se encuentran los millennials, una generación complicada de entender con respecto a los destinos turísticos de su elección.

Uno de los problemas a los que se enfrenta la industria turística es, que este segmento representa un cambio en los hábitos de consumo y en la relación con el cliente, lo cual también impacta en las tendencias, cuyo protagonismo es momentáneo, sus preferencias cambian de lo tradicional y cercano, a la cultura mexicana y a la inclusión de la tecnología en este sector, presentando a su vez decisiones cambiantes con un patrón en la conducta dentro de su círculo generacional. Para poder cumplir con las expectativas de esta generación con relación a un destino turístico, éste debe ofrecer los siguientes elementos: tecnología, hospedaje, transporte y amenidades, por mencionar algunos de los más conocidos.

Se han realizado estudios sobre destinos turísticos, turismo gastronómico, marketing turístico, el papel central del comportamiento del turista tradicional, así como de las estrategias utilizadas para la promoción de los destinos turísticos (Bigné, Andreu, Sánchez y Alvarado, 2008), siendo el vacío detectado en la literatura sobre el turista millennial, sus características y sus preferencias. Los resultados que se obtengan de esta investigación permitirán conocer los elementos que influyen en la toma de decisión del destino, para saber hacia dónde dirigir los esfuerzos mercadológicos y así atraer a este segmento a un destino turístico en específico, lo que conllevará al aumento de ofertas turísticas, como también a la gran competencia entre empresas del ramo.

\section{MATERIAL Y MÉTODO}

El presente trabajo se realizó bajo el enfoque cualitativo con método inductivo y de tipo explicativo, haciendo uso de las técnicas de revisión documental, tabla de contenidos y grupo de enfoque. Para este efecto, se realizó un grupo de enfoque con un guion de 10 preguntas orientadas hacia el descubrimiento de los medios virtuales, preferidos para búsqueda de información y destinos turísticos. La operatividad del constructo fue destino turístico y sus categorías: tradicional, moderno y sostenible. El grupo estuvo conformado por una selección 
MARKETING TURÍSTICO PARA MILLENNIALS.

intencional de 10 personas entre los 20 y 35 años de edad, así como de diversas áreas del conocimiento y del sector productivo.

\section{DESARROLLO}

El turismo es un fenómeno social, cultural y económico, que a lo largo del tiempo ha sido un promotor de la economía mundial, de la generación de empleo, de productos y de servicios, protección ambiental y preservación cultural a nivel internacional. Durante los últimos 60 años, se ha distinguido por tener un crecimiento interrumpido, reflejado en los ingresos por destinos de todo el mundo y categoría importante del comercio internacional de servicios; representa el $7 \%$ de las exportaciones mundiales de bienes y servicios y ocupa el tercer lugar como categoría mundial de exportación (OMT, 2017).

Las primeras definiciones del turismo eran muy amplias y ambiguas, actividad que involucra el desplazamiento de una persona por negocios o placer (McIntosh, Goeldner y Ritchie, 2001; OMT, 2007; SECTUR, 2018); lo que llevó a su vez que también se clasificara el término viajante. En la actualidad, existen tres tipos de viajantes definidos como: visitante, turista y excursionista (Acerenza, 2006).

El turismo ha cambiado en las últimas décadas, sus modificaciones se han visto afectadas por el desarrollo tecnológico, la conformación de nuevas identidades, las prácticas sociales, las formas de actuar, así como por los deseos y las costumbres que caracterizan al sujeto posmoderno, el cual lleva una vida cultural centrada en el consumo, con mayor dependencia de la tecnología, inmerso a nuevas formas de vincularse socialmente (López y Méndez, 2015).

Un elemento importante en la cultura posmoderna y que influye en la forma en que el sujeto construye los destinos turísticos, es el consumo, el cual se convierte en una forma del individuo para darle un sentido existencial a sus vidas. La posibilidad de adquirir objetos, de ser usuario de servicios, de habitar, aunque sea temporalmente ciertos lugares, de tener experiencias significativas, se vuelven necesidades que moldean prácticas sociales, las percepciones, las formas de producción, intercambio y apropiación de las mercancías (López y Méndez, 2015).

Un grupo de Expertos en Gestión de Destinos de la OMT, define el destino turístico como un espacio físico en el cual un turista está al menos una noche; donde se le ofertan productos, tales como servicios de apoyo, atracciones y recursos turísticos que pueden ser consumidos en un recorrido de ida y vuelta el mismo día (Barrado, 2004).

CORNELIO-VÁZQUEZ B. L., CUEVAS-ÁLVAREZ M. C. 


\section{MARKETING TURÍSTICO PARA MILLENNIALS.}

Entendiéndose entonces el destino turístico no solamente como un área geográfica, sino el conjunto de componentes que permiten el goce y disfrute de un área territorial, estos componentes hacen referencia a los servicios que se prestan dentro de esa zona, como lo es el atractivo turístico, las tiendas de souvenirs, los restaurantes, los sanitarios, los guías turísticos, por mencionar algunos.

Para poder dar promoción de esos destinos, el marketing turístico identifica los segmentos de mercado, promueve el desarrollo de productos turísticos y proporciona información sobre los productos ofertados (Trigueiro, 1999). El marketing turístico puede definirse entonces, como el conjunto de actividades con las que se facilita la el intercambio entre los diversos proveedores (Vaz, 2002,18). Por lo tanto, es necesario que el marketing turístico conozca las preferencias de los turistas y divisar el abanico de posibilidades que se puedan proporcionar (Barroso y Mota, 2010).

El marketing turístico requiere, para publicitar un producto turístico, de un análisis sistemático y permanente de las necesidades de los turistas y la comunidad local, así como una reflexión sobre su situación actual. Un análisis sobre la evolución de sus mercados e identificación de los diferentes productos, mercados o segmentos actuales y potenciales, con el objetivo de detectar amenazas y oportunidades (Camacho y Andrade, 2015).

Este tipo de investigación busca patrones en los grupos generacionales para aplicar una estrategia acorde a preferencias. "Un grupo comparte a lo largo de su historia un conjunto de experiencias formativas que los distinguen de sus predecesores" (Simón y Allard, 2007). Las vivencias comunes y no los rangos de edad son los que determinen a una generación y marca una diferencia importante en la evolución del estudio intergeneracional.

Actualmente, pueden distinguirse cuatro generaciones que conviven y comparten los espacios turísticos: los tradicionalistas, nacidos hasta 1945, los baby boomers, nacidos entre 1946 y 1963, la generación X, nacidos entre 1964 y 1979 y la generación Y, nacidos a partir de 1980 (González, 2011). Ésta última también denominada Generación del Milenio o Millennial, se distingue por buscar vivir bien el presente, realizar lo que les da placer, y no organizan su vida alrededor de lo que consideran un compromiso o atadura.

Además, se caracterizan por ser un consumidor crítico que utiliza el Internet para investigar y contrastar información y las redes sociales son un componente inseparable. Por consiguiente, 
MARKETING TURÍSTICO PARA MILLENNIALS.

están conscientes de que viven en un mundo globalizado y son jóvenes que han crecido en la abundancia y en la expansión económica (Gilibets, 2013).

Precisamente, porque buscan vivir el aquí y el ahora, no es un simple consumidor, sino un prosumidor, el cual debe divulgar sus experiencias en su mundo virtual como viajante. Los productos físicos pierden importancia ante las nuevas experiencias que les permiten obtener conocimientos para su desarrollo profesional, volviéndose consumidores sociales, y a diferencia de otros segmentos, posee mayor resistencia ante la crisis y desastres naturales (Canalis, 2013 y Excélsior, 2018) ya que viajar es una parte fundamental para su estilo de vida.

La estrecha relación entre millennials y turismo, ha convertido a esta generación en un reto para el marketing turístico, ya que se mueven mayoritariamente online a lo largo de todo el proceso: desde la búsqueda de destino, la comparación entre los proveedores de servicios y costos, hasta el momento de compartir su experiencia en redes sociales (Lostale, 2016).

En ese sentido, las redes sociales se utilizan de preferencia para búsqueda de información, donde se obtienen los siguientes datos, acorde a los autores: a) Facebook, b) Twitter, c) YouTube, d) Flicker y e) TripAdvisor (Filho y Tan, 2008; Torres, 2009; Safko y Brake, 2010; Barefoot y Szabo, 2010; Telles, 2010; Cruz Velozo y Soares, 2011; TripAdvisor, 2011).

Si bien, sus preferencias se extrapolan, los millennials buscan desde acercamiento a la cultura tradicional o a la modernidad; sin embargo, hay un segmento que prefiere una conjugación de ambas partes, a lo que se le denomina como Destino Turístico Inteligente (DTI) el cual surge del concepto ciudades inteligentes o Smart Cities (Boes, Buhalis, y Inversini, 2015; Gretzel et al., 2015; Zhu, Zhang y Li, 2014).

Acorde con la OMT (Dyma, 2014), este sector de la población realiza más de 190 millones de viajes por año, lo que equivale a una economía de 165 mil millones de dólares; a diferencia de otros segmentos, éste es el que más crecimiento ha tenido a nivel global, asimismo conlleva a crear oportunidades, pero también a asumir ciertos retos ante estos nuevos turistas. A continuación, se presenta una tabla con las características de este segmento, las oportunidades y retos a lo que se tiene que enfrentar el marketing turístico actual. 


\section{Tabla 1}

Oportunidades y retos ante la nueva generación de turistas

\begin{tabular}{|c|c|c|}
\hline $\begin{array}{l}\text { Características de los } \\
\text { millennials }\end{array}$ & Oportunidades & Retos \\
\hline $\begin{array}{l}\text { Mayor movilidad y } \\
\text { viajeros más } \\
\text { experimentados. }\end{array}$ & $\begin{array}{l}\text { - Mayor número de viajes } \\
\text { puede significar un alivio } \\
\text { para los } \\
\text { estancados. }\end{array}$ & $\begin{array}{l}\text { - Turistas con r altas } \\
\begin{array}{l}\text { expectativas sobre los } \\
\text { destinos. }\end{array}\end{array}$ \\
\hline $\begin{array}{l}\text { Interés creciente en } \\
\text { viajes de largo recorrido. }\end{array}$ & $\begin{array}{l}\text { - Capacitación de turistas en } \\
\text { otros continentes. } \\
\text { - Los viajes de larga distancia } \\
\text { se convierten en "ritos de } \\
\text { paso" durante la juventud. }\end{array}$ & $\begin{array}{l}\text { - Mayor competencia a } \\
\text { nivel global. } \\
\text { - Presión sobre los destinos } \\
\text { y viajes domésticos. }\end{array}$ \\
\hline $\begin{array}{l}\text { Interesados en viajes } \\
\text { domésticos. }\end{array}$ & $\begin{array}{l}\text { - Vacaciones de corta } \\
\text { estancia accesibles por } \\
\text { coche o avión. } \\
\text { - Vacaciones de media y larga } \\
\text { estancia para familias } \\
\text { jóvenes con hijos. }\end{array}$ & $\begin{array}{l}\text { - Adaptar los destinos a las } \\
\text { necesidades de los } \\
\text { millennials. } \\
\text { - Abastecimiento para unos } \\
\text { turistas cada vez más } \\
\text { diversos. }\end{array}$ \\
\hline $\begin{array}{l}\text { Mayor diversidad de } \\
\text { estructuras familiares. }\end{array}$ & $\begin{array}{l}\text { - El marketing actual permite } \\
\text { llegar a mayores segmentos } \\
\text { de mercado (solteros, } \\
\text { parejas, parejas con hijos...). }\end{array}$ & $\begin{array}{l}\text { - Dificultad para anticipar } \\
\text { las estructuras familiares } \\
\text { del futuro debido a la } \\
\text { incertidumbre de la } \\
\text { generación millennial. }\end{array}$ \\
\hline $\begin{array}{l}\text { Familiarización con los } \\
\text { medios digitales. }\end{array}$ & 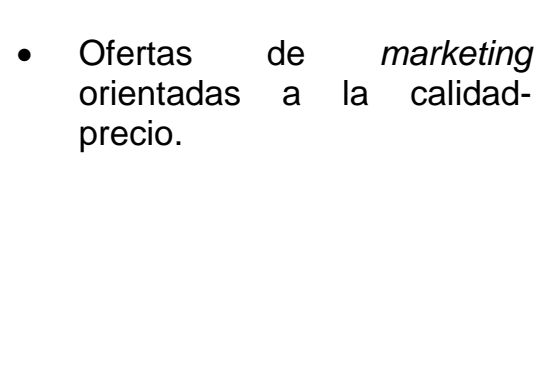 & $\begin{array}{l}\text { - La oferta turística debe } \\
\text { adaptarse a los medios } \\
\text { digitales para } \\
\text { comunicarse con todo tipo } \\
\text { de turistas. } \\
\text { - La mayor transparencia } \\
\text { que permiten las redes } \\
\text { obliga a que el producto } \\
\text { esté bien definido. }\end{array}$ \\
\hline $\begin{array}{l}\text { Curiosidad por destinos } \\
\text { nuevos y genuinos. }\end{array}$ & $\begin{array}{l}\text { - Oportunidades para destinos } \\
\text { emergentes y productos } \\
\text { innovadores. }\end{array}$ & $\begin{array}{l}\text { - Dificultad para mantener a } \\
\text { turistas recurrentes en un } \\
\text { destino. } \\
\text { - Necesidad de proveer } \\
\text { experiencias únicas y } \\
\text { "frescas" para atraer a } \\
\text { turistas internacionales. }\end{array}$ \\
\hline
\end{tabular}

Fuente: Fernández et al $(2017,141)$.

CORNELIO-VÁZQUEZ B. L., CUEVAS-ÁLVAREZ M. C. 


\section{MARKETING TURÍSTICO PARA MILLENNIALS.}

\section{HALLAZGOS}

Un área de oportunidad que se encuentra en la revisión de la literatura, es el DTI. Este es un destino que tiene un proyecto definido, con visión de desarrollo sostenible del territorio, con alto grado de innovación mediante uso considerable de tecnología y esfuerzos dirigidos a mejorar la calidad de la estancia de los turistas, agregando así oferta de valor, satisfacción y experiencias a los turistas millennials.

Como el caso de El Hierro en España, la primera isla inteligente por contar con autosuficiencia energética y menor contaminación, al generar energía con la basura, integración de antenas, placas solares en el paisaje y sustitución del ladrillo por piedra volcánica.

Otro caso es Singapore Smart City, un desarrollo de comunidades inteligentes que ofertará teléfonos de emergencia e información con geo-posicionamiento del turista, para indicar comisarías u hospitales cercanos o eventos que estén sucediendo al momento.

El Pueblo Mágico de Tequila en Jalisco, México se ha posicionado como el primer destino inteligente del país, a través del uso del Big Data en sus puntos de venta y búsquedas online permite, a través de sensores de calor, identificar concentraciones de personas y generar estadísticas de turistas visitantes, preferencias y consumos con lo que se han afinado las ofertas para brindar una propuesta de valor.

Dentro de los beneficios de un DTI acorde a SEGITTUR (2013) son:

a) La existencia de factores diferenciales en el desarrollo de las infraestructuras del destino convirtiéndolo en atractivo e innovador.

b) Se incremente la calidad de vida del residente local.

c) Aumente la competitividad de las empresas ubicadas en él con sistemas que facilitan la gestión de la información y facilitan la calidad de la visita.

En lo que respecta a los informantes, no se mostraron interesados ni entusiasmados en destinos turísticos considerados por ellos mismos típicos a la temporada: destinos de playa en semana santa o verano y destinos con clima frío para navidad o invierno. También, agregaron que los destinos turísticos carecen de servicios tecnológicos que se consideran básicos en 
MARKETING TURÍSTICO PARA MILLENNIALS.

otros países: servicio de WiFi gratuito en los hoteles o restaurantes, solamente cuando deberían tener este servicio, también en los parques o plazas cívicas.

Consideran que debería haber acceso a información histórica o para emergencias de forma más dinámica. En caso de perder su celular, quedarse sin batería o peor aún sin datos, no hay otras maneras de obtener información para desplazarse, asistir a algún evento o concierto cerca de ellos, si no se enteran a través de los medios comunes: radio, televisión o impresos.

Los informantes se mostraron interesados en la tercera opción de DTI e indicaron no conocer alguno, ni recibir publicidad al respecto o haber visto algún banner o imagen que los promocione en ninguna de las cuentas sociales que utilizan. Los informantes de manera inmediata comenzaron a realizar una búsqueda con alguna proveedora de servicios que les indicara precios sobre los destinos mencionados durante el grupo de enfoque, sin respuesta alguna.

Los informantes indicaron que no les importaría pagar por el servicio, aunque su costo fuera elevado, el vivir por unos instantes en un lugar con calidad de primer mundo, sería una experiencia inigualable e irrepetible, que mantendrían en su memoria indefinidamente, como también en sus redes sociales.

Finalmente, ellos recomiendan que las empresas proveedoras de servicios enfoquen sus esfuerzos a la promoción y publicidad de estos lugares, además de que otros destinos tomen esta iniciativa que sin duda permitirá una mayor derrama económica y que haya otro nivel de competencia de un destino a otro.

\section{CONCLUSIONES}

México cuyo atractivo turístico radica en su gran biodiversidad y en sus culturas milenarias, tiene una gran variedad de destinos que ofrecer, desde una ciudad modernizada con rascacielos hasta un pueblo indígena con ruinas prehispánicas. México cuenta con los servicios necesarios para una estancia cómoda durante el tiempo que permanezcan en el destino de su elección.

Los millennials son muy importantes, porque son los viajeros pioneros que descubren y ponen de moda nuevos destinos; para ellos el turismo convencional de visitar museos y monumentos 
MARKETING TURÍSTICO PARA MILLENNIALS.

ya no es suficiente, las nuevas generaciones piden un contacto más cercano con la cultura, las experiencias y las personas del destino que visitan.

Además, aprecian pasear y perderse por la ciudad, hablando con gente local y descubriendo por sí mismos la esencia de la localidad, pueblo, estado o país que visiten. Es por ello, que es necesario dirigir los esfuerzos mercadológicos hacia los elementos que influyen en la toma de decisión de los millennials: desde un sitio web, las amenidades, la transportación, el hospedaje, los alimentos, pero sobretodo las experiencias que el destino ofrece, que otros servicios y operadores turísticos no ofrezcan.

En la actualidad, las tendencias tienen una durabilidad menor a la que existía en años anteriores, lo que conlleva indudablemente al aumento de ofertas de experiencias turísticas por parte de las empresas del ramo turístico.

Los DTI son la propuesta u opción lógica y es el reto actual para el marketing turístico. La promoción de productos y comunidades que garanticen la preservación del patrimonio cultural y natural, así como la ampliación de los beneficios y amenidades para que esta industria turística continúe generando ingresos a la economía mexicana y con impacto en la economía global.

\section{REFERENCIAS BIBLIOGRÁFICAS}

A. M. U. (5 de abril de 2018). Millennials impulsan la industria turística nacional e internacional. Excélsior. Recuperado de https://www.excelsior.com.mx/nacional/millennials-impulsan-laindustria-turistica-nacional-e-internacional/1230763\#view-2

Acerenza, M. (2006). Conceptualización, origen y evolución del Turismo. México: Trillas.

Aires, G. y Nicolau, K. (2010). Marketing turístico internacional. La Marca Brasil. Estudios y Perspectivas en Turismo, 19(2), 241-267.

Barefoot, D. y Szabo, J. (2010). Friends with benefits: A social media-marketing handbook. San Francisco: No Starch Press. 


\section{MARKETING TURÍSTICO PARA MILLENNIALS.}

Barrado, D. (2004). El concepto de destino turístico: Una aproximación geográfico-territorial. Estudios Turísticos, 1(160), 45-68.

Bigné, J., Andreu, L., Sánchez, I. y Alvarado, A. (2008). Investigación internacional en marketing turístico: análisis de contenido sobre temas y metodologías. PASOS Revista de Turismo y Patrimonio Cultural, 6(3), 391-398.

Boes, K., Buhalis, D. y Inversini, A. (2015). Conceptualising smart tourism destination dimensions. En Z. Xiang y I. Tussyadiah (Eds.), Information and Communication Technologies in Tourism (391-403) Suiza: Springer International Publishing.

Camacho, M. y Andrade, J. (2015). Mercadotecnia en perspectiva: reflexiones y casos. México: Trillas.

Canalis, X. (2013). Millennials: el target de moda para el turismo. Recuperado de https://issuu.com/hosteltur_2013/docs/hosteltur_232-millennials_el_target

Cruz, G., Velozo, T. y Soares, A. E. F. (2011). Twitter, Youtube e innovación en la promoción turística online: Análisis de las estrategias del Ministerio de Turismo de Brasil. Estudios y Perspectivas en Turismo. 20(3), 627-642.

Dyma, A. (2014). Un análisis de la oferta turística en la ciudad de Málaga para el turismo joven; nuevas tendencias de consumo. (Tesis de pregrado Universidad de Málaga, España). Recuperado de https://riuma.uma.es/xmlui/bitstream/handle/10630/7979/ALINA\%20DYMA_ TFG.pdf?sequence=1

De la Madrid, E. (5 de octubre de 2018). Destinos Turísticos Inteligentes. Excélsior. Recuperado de https://www.eluniversal.com.mx/articulo/enrique-de-la-madrid/nacion/destinos-turisticosinteligentes

Fernández, A., López, J., Moreno, L., Perles, J., Ramón, A. y Such, M. (2017). Innovación y destinos inteligentes: oportunidad para el know how turístico español. Estrategia e internacionalización de la empresa turística, 894(1), 137-150.

Filho, L. y Tan, F. (2008). An overview on user-generated content and the empowerment of online travelers. Revista da FARN, Natal, 7(2), 17-30. 


\section{MARKETING TURÍSTICO PARA MILLENNIALS.}

Gilibets, L. (2013). Los millennials: la nueva generación de consumidores 2.0. Recuperado de: http://comunidad.iebschool.com/iebs/marketing-digital/millennials/

González, R. (2011). La incorporación de la Generación Y al mercado laboral. El caso de una Entidad Financiera de la ciudad de Resistencia. Palermo Business Review, 1(5), 67-93.

Gretzel, U., Sigala, M., Xiang, Z. y Koo, C. (2015). Smart tourism: foundations and developments. Electronic Markets, 25(3), 179-188.

Hosteltur (2013). Millennials el target de moda del turismo. Hosteltur, 1(232), 3-17. Recuperado de https://www.hosteltur.com/119900_millennials-target-moda-turismo.html

Huerta, G. (s.f). El turismo millennial en México. México: Entorno Turístico. Recuperado de https://www.entornoturistico.com/el-turismo-millennial-en-mexico/

Lostale, E. (2016). Marketing turístico para millennials: el gran desafío. Recuperado de https://www.kanlli.com/sectores/marketing-turistico-millennials-gran-desafio/

López, L. y Méndez, E. (2015). El turismo en tiempos del ciberespacio. Contexto. Revista de la Facultad de Arquitectura de la Universidad Autónoma de Nuevo León, 9(10), 33-42.

Mclntosh, R., Goeldner, C. y Ritchie, J. (2001). Turismo: planeación, administración y perspectiva. México: Limusa Wiley.

OMT. Organización Mundial de Turismo. (2007). Entender el turismo: Glosario Básico. Recuperado de http://media.unwto.org/es/content/entender-el-turismo-glosario-basico

OMT. Organización Mundial del Turismo. (2017). Panorama OMT del turismo internacional. UNWTO Publications. Recuperado de https://www.eunwto.org/doi/pdf/10.18111/9789284419043

Safko, L. y Brake, D. K. (2010). La biblia de la red social: tácticas, herramientas y estrategias para construir y transformar los negocios. São Paulo: Blucher.

SECTUR. Secretaría de Turismo. (2018). Definiciones del turismo. Recuperado de https://www.gob.mx/sectur/ 
MARKETING TURÍSTICO PARA MILLENNIALS.

SEGITTUR. Sociedad Estatal para la Gestión de la Innovación y las Tecnologías Turísticas (s/f). Destino Turísticos Inteligentes. SEGITTUR: España. Recuperado de cf.cdn.unwto.org/sites/.../3_baldomero_leon_navarro_informe_final_dti_marbella.pdf

Simón, C. y Allard, G. (2007). Generación "Y" y mercado laboral: Modelo de gestión de recursos humanos para los jóvenes profesionales. España: Instituto de Empresa Business School.

Telles, A. (2010). La revolución de las redes sociales. São Paulo: M. Books.

Torres, C. (2009). La biblia del marketing digital: todo lo que usted quería saber de marketing y publicidad en internet y no tenía a quien preguntar. São Paulo: Novatec.

Trigueiro, C. (1999). Marketing e turismo: como planejar e administrar o marketing turístico para uma localidade. Rio de Janeiro: Qualitymark Editora.

Tripadvisor. (2011). Sobre TripAdvisor ${ }^{\circledR}$ Media Group. Acceso el: 27 de mayo 2018. Recuperado de http://www.tripadvisor.com.br/pages/about_us.html.

Vaz, G. (2002). Marketing turístico: receptivo e emissivo: um roteiro estratégico para projetos mercadológicos públicos e privados. São Paulo: Pioneira Thomson Learning. 